\title{
Phase-field simulation of solidification dendritic segregation in Ti-45Al alloy
}

\author{
${ }^{*}$ Yu-tuo Zhang ${ }^{1}$, Yun $\mathrm{Chi}^{2}$, and Chun-qing Hu ${ }^{1}$ \\ 1. School of Materials Science and Engineering, Shenyang Ligong University, Shenyang 110159, China; \\ 2. Liaoning Academy of Governance, Shenyang 110161, China
}

\begin{abstract}
The microstructures and mechanical properties of TiAl alloys are directly linked to micro-segregation which cannot be avoided during solidification. So a thorough understanding of the micro-segregation should be a great help to further enhance the mechanical properties of the cast products. Theoretical analysis and experiments have been used to predict the micro-segregation, but it is very difficult to observe and determine the dendritic segregation in the micro region. Phase-field method has been employed for the simulation of dendritic growth. However, due to the complicated quasi-sub regular solution model for Ti-45Al(at.\%) alloy, the classic phase-field models have difficulty to deal with the free energy. In this work, a phase-field model by linking thermodynamic calculation was used to simulate solidification dendritic segregation of Ti-45Al alloy for Liquid $\rightarrow$ Liquid $+\beta(T i)$. The free energies of solid phase and liquid phase for Ti-45Al alloy were calculated by Thermo-Calc and then coupled with the phase-field equations. The simulation results show the dendritic morphology and Al content variations between liquid and growing solid phase for Ti-45Al alloy. With the growth of the $\beta(\mathrm{Ti})$, dendritic segregation is formed in the liquid and solid phases due to the solute partitioning and rejection into the liquid. As a result, the dendrite arms are depleted of Al element, while the inter-dendrites are enriched. The dendritic tip growth velocity decreases with the progress of solidification, whereas the segregation ratio increases.
\end{abstract}

Key words: phase-field model; dendritic segregation; Ti-45Al; free energy; isothermal solidification

CLC numbers: TP391.92 Document code: A Article ID: 1672-6421(2017)03-184-04

$\mathrm{T}^{\mathrm{n}}$ he micro-segregation in TiAl alloy cannot be avoided during casting and leads to microstructural deviation. Dendritic segregation in TiAl alloy solidification results in the formation of secondary phases, cracks, and other problems. Thus, a thorough understanding of the solidification segregation should be a great help to further enhance the mechanical properties of the cast products. Therefore, it is required to predict segregation of solute with dendritic solidification ${ }^{[1-4]}$. Theoretical analysis and experiments have been used to predict the micro-segregation, but it is very difficult to observe and determine the dendritic segregation in the micro region.

The phase-field method is a powerful computational approach to simulate the evolution of dendritic morphology and micro-segregation during solidification.

\footnotetext{
*Yu-tuo Zhang

Female, born in 1966, Ph.D., professor. Her research mainly focuses on metal processing and computer simulation of solidification. She has published more than 50 papers.

E-mail: ytzhang@imr.ac.cn
}

Received: 2016-11-30; Accepted: 2017-03-20

The phase field model was originally proposed for simulating the dendrite growth in undercooled pure metals and has been extended to the solidification of alloys. The first model for alloy solidification which was proposed by Wheeler et al. ${ }^{[5]}$ is known as the classic WBM model. The model has been the most widely used in ideal solutions of binary alloys corresponding to the simple lens type phase diagrams and is derived in a thermodynamically consistent way ${ }^{[6-8]}$. In the model, any point within the interfacial region is treated as an interpolation between solid and liquid phases with the same composition. Kim et al. ${ }^{[9]}$ proposed a new phase field model for binary alloys, named KKS model which is similar to the WBM model, but has a different definition of the free energy for the interfacial region with the different compositions. The KKS model has been widely used to simulate the dendritic growth in dilute solutions of alloys corresponding to the linearization of the phase diagram lines ${ }^{[9]}$.

However, due to the complicated quasi-sub regular solution model for Ti-45Al(at.\%) alloy, the classic WBM phase-field model is hardly able to deal with the free energy, and the KKS model also has difficulty in 
constructing the free energy densities of solid and liquid. In order to overcome this limitation, a phase-field model coupling with Calphad thermodynamic calculation has been adopted by Guo et al. ${ }^{[10,11]}$ for the simulation of TiAl alloy directional solidification. Based on the thermodynamic calculation method ${ }^{[12]}$, in this work, a phase-field model by linking thermodynamic calculation was used to simulate solidification dendritic segregation of Ti$45 \mathrm{Al}$ alloy for Liquid $\rightarrow$ Liquid $+\beta(\mathrm{Ti})$. The free energies of solid phase and liquid phase for Ti-45Al alloy are calculated by Thermo-Calc and then coupled with the phase-field equations. Equilibrium partition coefficient $k_{0}$ and the degree of segregation $\left(1-k_{0}\right)$ for Ti-Al alloy are also calculated. The segregation ratio of $\mathrm{Al}$ and dendrite tip growth velocity with the development of solidification for Ti-45Al alloy at $1,780 \mathrm{~K}$ are also discussed.

\section{Phase-field model}

\subsection{Governing equations}

The phase-field model used in this study can be found in reference ${ }^{[13]}$ for details of the formulation. The governing equations can be written as:

$$
\begin{aligned}
& \frac{\partial \phi}{\partial t}=M_{\phi}\left(\varepsilon^{2} \nabla^{2} \phi-f_{\phi}\right) \\
& \frac{\partial c}{\partial t}=\nabla\left[M_{c} c(1-c) \nabla f_{c}\right]
\end{aligned}
$$

where $\phi$ is the phase field, $\phi=0$ represents liquid, $\phi=1$ represents solid and $0<\phi<1$ at the interface. $c$ is the solute composition, $\varepsilon$ is the gradient energy coefficient, $\varepsilon^{2}=6 \sigma_{A} \delta_{A}$. The free energy density of the system $f(c, \phi, T)$ can be described as the following:

$$
f(c, \phi, T)=\left[(1-c) W_{A}+c W_{B}\right] g(\phi)+[1-p(\phi)] f^{S}(c, T)+p(\phi) f^{L}(c, T)
$$

All the parameters needed in this model can be calculated as follows:

$$
\begin{gathered}
M_{\phi}=(1-c) M_{\phi}^{A}+c M_{\phi}^{B} \\
M_{c}=\frac{V_{m}}{R T}\left\{p(\phi) D_{S}+[1-p(\phi)] D_{L}\right\} \\
g(\phi)=\phi^{2}\left(1-\phi^{2}\right) \\
p(\phi)=\phi^{3}\left(1-15 \phi+6 \phi^{2}\right)
\end{gathered}
$$

where $T$ is the temperature, $M_{\phi}, M_{c}, W_{A}, W_{B}$ are phasefield parameters, $W_{A}=\frac{3 \sigma_{A}}{\delta_{A}}, \quad M_{\phi}^{A}=\frac{\beta_{A} T_{m}^{A}}{6 \sigma_{A} L^{A}}, \quad W_{B}=\frac{3 \sigma_{B}}{\delta_{B}}$ $M_{\phi}^{B}=\frac{\beta_{B} T_{m}^{B}}{6 \sigma_{B} L^{B}}, f^{S}(c, T)$ and $f^{L}(c, T)$ are the free energies of solid and liquid phases, respectively, which are derived from the thermodynamic calculation of Ti-Al alloy by Thermo-Calc. $V_{\mathrm{m}}$ is the molar volume of the system. $\delta_{\mathrm{A}}, \sigma_{\mathrm{A}}$, and , $\delta_{\mathrm{B}}, \sigma_{\mathrm{B}}$ are the interface thickness and interfacial energy between solid phase and liquid phase for pure metal A and B, respectively. $L^{A}, T_{m}^{A}$ and $L^{B}, T_{m}^{B}$ are the latent heat and melting point for pure metal A and $\mathrm{B}$, respectively, here $\mathrm{A}$ is $\mathrm{Ti}$, and $\mathrm{B}$ is $\mathrm{Al}$. $D_{\mathrm{L}}$ and $D_{\mathrm{S}}$ are the diffusion coefficients in the bulk liquid and solid phases. $R$ is the gas constant. In simulations, the grid size was $\Delta x=\Delta y=10 \mathrm{~nm}$ and a grid system of $1,200 \times 1,200$ was used. The thermodynamic data are from the Thermo-Calc and other materials parameters are as follows ${ }^{[10]}: D_{\mathrm{L}}=2.8 \times 10^{-6}$ $\mathrm{cm}^{2} \cdot \mathrm{s}^{-1}, D_{\mathrm{s}}=3 \times 10^{-9} \mathrm{~cm}^{2} \cdot \mathrm{s}^{-1}, V_{\mathrm{m}}=2.34 \times 10^{-5} \mathrm{~m}^{3} \cdot \mathrm{mole}^{-1}, \beta_{\mathrm{Ti}}=0.387$ $\mathrm{cm} \cdot(\mathrm{K} \cdot \mathrm{s})^{-1}, \beta_{\mathrm{Al}}=0.441 \mathrm{~cm} \cdot(\mathrm{K} \cdot \mathrm{s})^{-1}, \sigma_{\mathrm{Ti}}=3.45 \times 10^{-5} \mathrm{~J} \cdot \mathrm{cm}^{-2}$, $\sigma_{\mathrm{Al}}=1.902 \times 10^{-5} \mathrm{~J} \cdot \mathrm{cm}^{-2}$.

\subsection{Free energy functional of Ti-Al alloy}

The driving force for phase transformation is defined by the chemical free energy, which depends on the temperature and concentration. An important part of a phase-field model is the free energy functional. The free energies of solid and liquid of Ti-45Al are obtained from the thermodynamic calculation by Thermo-Calc and then in conjunction with the phase-field equations. The curves for the free energies of the liquid and solid phases for Ti-Al alloy at 1,780 $\mathrm{K}$ are shown in Fig.1.

The free energy functional of liquid phase and solid phase for Ti-Al alloy at $1,780 \mathrm{~K}$ can be obtained by polynomial fitting, which can be written as follows:

$$
\begin{aligned}
& f^{S}(c, T)=100595 c^{4}-186578 c^{3}+239249 c^{2}-143703 c+4096 \\
& f^{L}(c, T)=-92793 c^{4}+175725 c^{3}-38749 c^{2}-54405 c-4617.5
\end{aligned}
$$

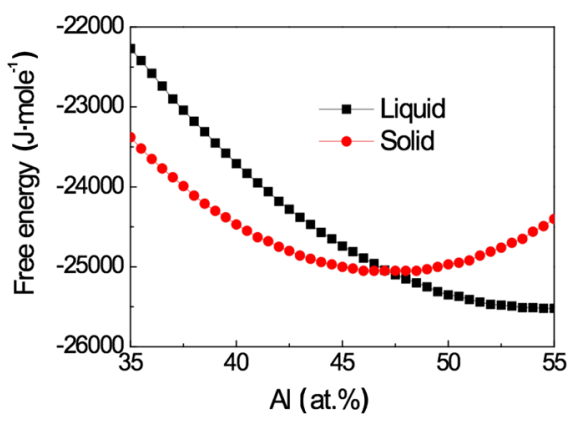

Fig. 1: Free energy of liquid and solid phases for Ti-Al alloy

\section{Analysis of solidification segregation of $\mathrm{Ti}-45 \mathrm{Al}$ alloy}

\subsection{Phase diagram calculation}

Figure 2 shows the part of the Ti-Al binary phase diagram which is calculated by Thermo-Calc. Ti-45Al alloy at $1,780 \mathrm{~K}$ for liquid-solid phase transition should lead to the following solidification sequence: Liquid $\rightarrow$ Liquid $+\beta(\mathrm{Ti})$. There is a wide range of solidification for Ti-45Al alloy and the calculated solidification temperature interval between the solidus and liquidus is $82 \mathrm{~K}$. The wider the temperature range of solidification of an alloy, the greater the development of segregation. 


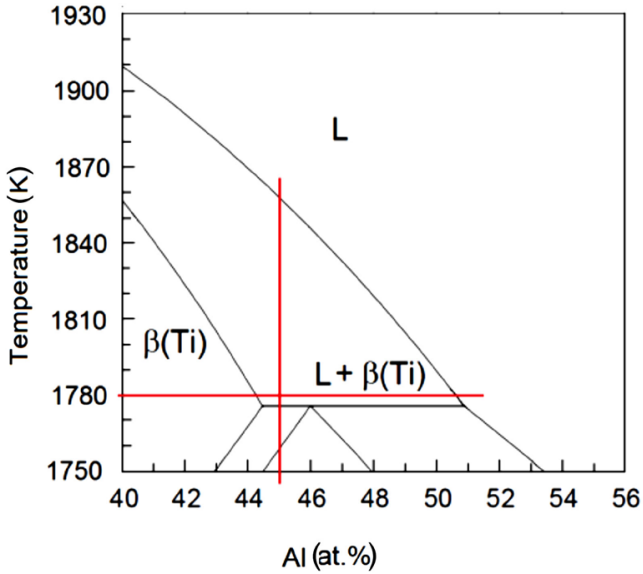

Fig. 2: Part of Ti-Al binary phase diagram calculated by Thermo-Calc

\subsection{Equilibrium partition coefficient}

The partition coefficient of solute element between liquid phase and solid phase is in relation to the formation of a solidification structure and the development of segregation. The equilibrium partition coefficient $k_{0}$ is a key parameter in the research of solute redistribution during solidification and plays an important role in the formation of micro-segregation. The equilibrium coefficient $k_{0}$ is defined as the ratio of the concentration of solute in the solid $\mathrm{C}_{\mathrm{S}}$ to that in the liquid $\mathrm{C}_{\mathrm{L}}$, when equilibrium exists between the solid and liquid phases at a given temperature. The equilibrium partition coefficient $k_{0}$ can be calculated to utilize liquidus and solidus curves directly from phase diagram, as shown in Fig. 3. Figure 4 shows the calculated equilibrium partition coefficient $k_{0}$ and the degree of segregation $\left(1-k_{0}\right)$ for Ti-Al alloy between solid and liquid phases with temperature. The partition coefficient changes at different temperatures and it increases with the increasing temperature. The $k_{0}$ for Ti- $45 \mathrm{Al}$ alloy is 0.874 at $1,780 \mathrm{~K}$. The degree of segregation of element in the alloy can be expressed by $\left(1-k_{0}\right)$. The larger the value of $\left(1-k_{0}\right)$, the more severe the segregation of $\mathrm{Al}$ element in the Ti-Al alloy solidification.

\section{Simulation of solidification dendritic segregation}

Figure 5 presents the simulated solute maps for Ti-45Al alloy during dendritic solidification at 1,780 $\mathrm{K}$ with (a), (b), (c) and (d) showing the dendritic segregation at $0.01 \mathrm{~ms}, 1.6 \mathrm{~ms}, 2.5$ $\mathrm{ms}$ and $5.0 \mathrm{~ms}$, respectively; (e), (f), (g) and (h) showing the Al concentration distribution along the horizontal dendrite arms from the center of the dendrites as the red straight line of the dendrite primary trunk along the dendrite axis direction. The primary phase is $\beta(\mathrm{Ti})$ in $\mathrm{Ti}-45 \mathrm{Al}$ with solidification dendritic microstructure. The chemical composition in the $\beta$ (Ti) dendrite trunk differs from that in the inter-dendritic zone. The $\mathrm{Al}$ content in the $\beta(\mathrm{Ti})$ phase varies and the first solidified solid has the lowest $\mathrm{Al}$ content.

Once nucleation occurs, subsequent growth of the solid is governed by solute diffusion. In the dendritic solidification

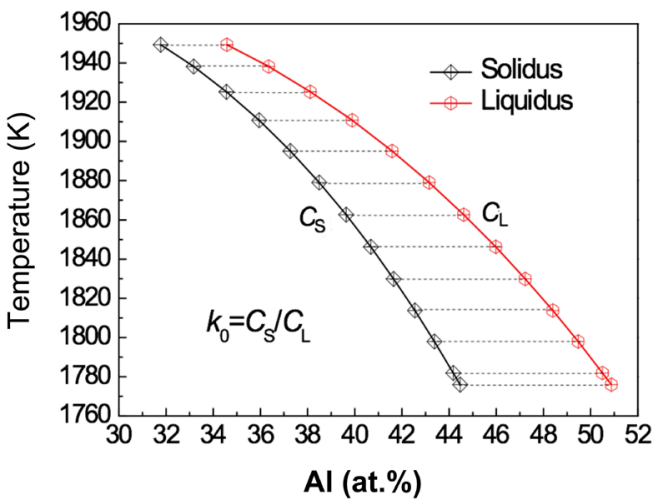

Fig. 3: Definition of partition coefficient with temperatures

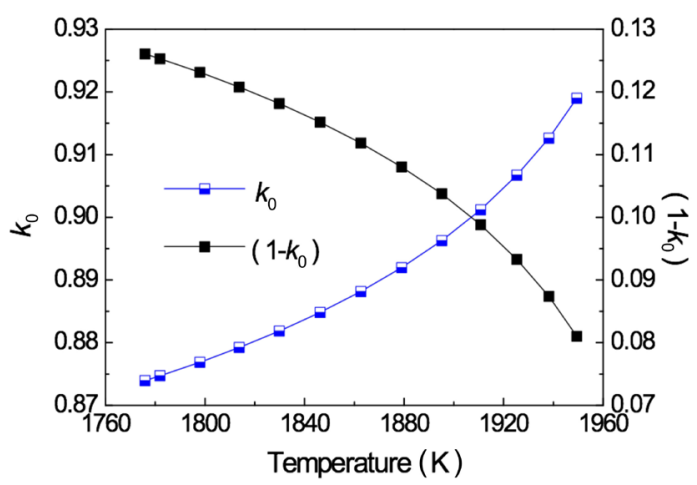

Fig. 4: Calculated $k_{0}$ and $\left(1-k_{0}\right)$ of Ti-Al alloy with temperature

process, solute diffusion in the liquid is usually sufficient, whereas in the solid it is limited diffusion. Ti is segregated into the $\beta(\mathrm{Ti})$ dendrite and $\mathrm{Al}$ is rejected into the liquid between dendrite arms. It can also be seen that the $\mathrm{Al}$ concentration fluctuates in the dendrite primary trunk and $\mathrm{Al}$ content is less than $45 \mathrm{at} . \%$, but there is solute enrichment in front of the solidliquid interface and $\mathrm{Al}$ content is more than 46at.\%, as can be seen from Fig. 5(f)-(h). The Al elements precipitated from the solid phase as a consequence of the solute redistribution during the solidification can not diffuse effectively, leading to the $\mathrm{Al}$ content gradient in the diffusive moving solid-liquid interface with a certain thickness. The dendritic tip growth velocity exceeds the diffusion rate of $\mathrm{Al}$ elements, and resulting in the phenomenon of $\mathrm{Al}$ content fluctuations along the dendrite axis direction. As the solidification proceeds, the rejection of $\mathrm{Al}$ element by the solid enriches in the solid/liquid interface as a result of the enrichment of solutes in the liquid phase, especially in liquid region between the dendritic arms.

Micro-segregation is a common phenomenon in dendritic solidification, which is usually considered as a solidification defect. The segregation ratio $S_{\mathrm{R}}$ is the ratio of the maximum Al content $\mathrm{C}_{\max }$ in inter-dendritic zone to the minimum $\mathrm{Al}$ content $\mathrm{C}_{\min }$ in dendritic trunk, $S_{\mathrm{R}}=\mathrm{C}_{\max } / \mathrm{C}_{\min }$. It can be used for the evaluation of dendritic segregation.

Figure 6 presents the segregation ratio of $\mathrm{Al}$ and dendrite tip growth velocity with the development of solidification for Ti$45 \mathrm{Al}$ alloy at $1,780 \mathrm{~K}$. The segregation ratio of $\mathrm{Al}$ increases with increasing solidification time, whereas the tip velocity decreases with time. The dendrite tip velocity starts from a large value, and 

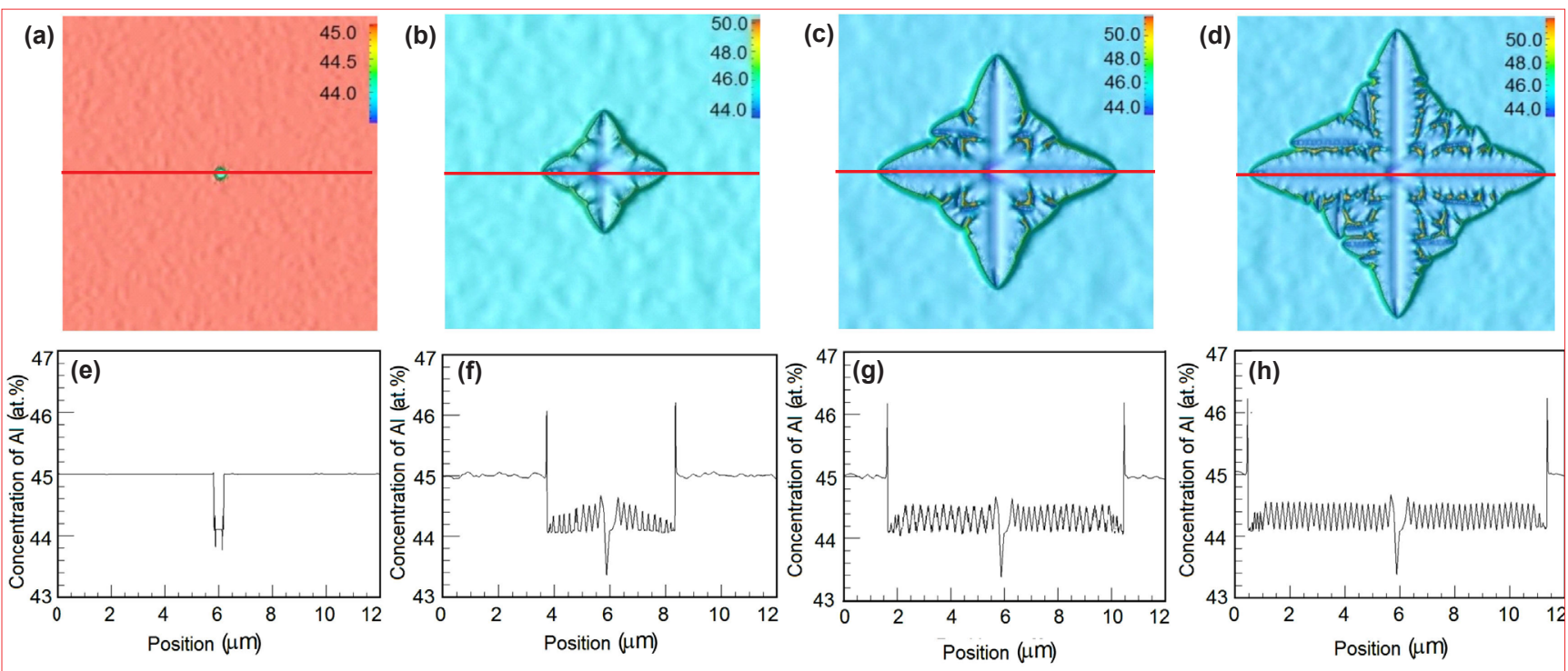

Fig. 5: Simulated solute maps for Ti-45Al alloy during dendritic solidification at 1,780 K. The first row (a)- (d) shows the dendritic segregation at $0.01 \mathrm{~ms}, 1.6 \mathrm{~ms}, 2.5 \mathrm{~ms}$ and $5.0 \mathrm{~ms}$, respectively. Second raw (e)-(h) shows the Al concentration distribution along the red straight line of the dendrite primary trunk

then slows down in the later stage of solidification as a result of the enrichment of solutes in the liquid phase.

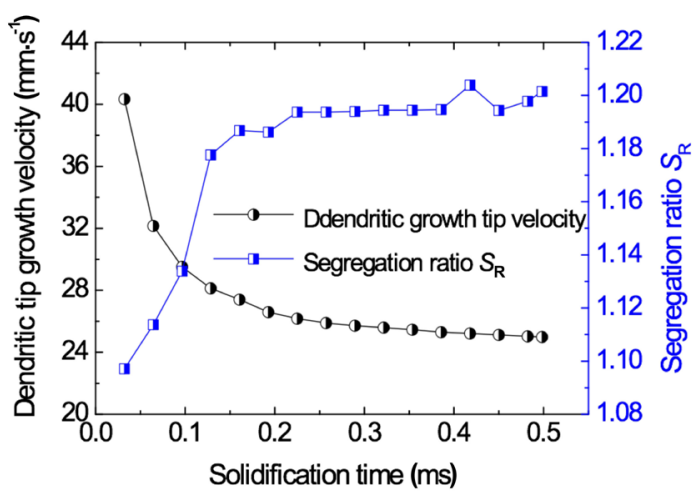

Fig. 6: Segregation ratio and dendritic tip growth velocity with time for Ti-45Al alloy at $1,780 \mathrm{~K}$

\section{Conclusions}

(1) The phase-field model coupled with free energy calculation by Thermo-Calc is successfully used to simulate the dendritic segregation of Ti-45Al alloy. The calculated partition coefficient $k_{0}$ of Ti-Al alloy increases with temperature increasing. The $k_{0}$ for Ti-45Al alloy at $1,780 \mathrm{~K}$ is 0.874 .

(2) The solute element $\mathrm{Al}$ redistribution in Ti-45Al alloy at $1,780 \mathrm{~K}$ solidification process results in dendritic segregation. $\mathrm{Al}$ concentration fluctuates in the dendrite primary trunk along the dendrite axis direction and $\mathrm{Al}$ content is less than $45 \mathrm{at} . \%$. There is solute enrichment in front of the solid-liquid interface and $\mathrm{Al}$ content is more than 46 at. $\%$.

(3) The segregation ratio of $\mathrm{Al}$ increases with the progress of solidification, whereas the dendritic tip growth velocity decreases.

\section{References}

[1] Daloz D, Hecht U, Zollinger J, et al. Micro-segregation, macrosegregation and related phase transformations in TiAl alloys. Intermetallics, 2011, 19: 749-756.

[2] Kurz W, and Fisher D J. Fundamentals of Solidification. Fourth revised edition, Trans Tech Publications Ltd, Switzerland, 1998.

[3] Himemiya, Toshiaki and Umeda, Takateru. Solute redistribution model of dendritic solidification considering diffusion in both the liquid and solid phases. ISIJ International, 199838 (7): 730-738.

[4] Won Young-Mok and Thomas B G. Simple model of microsegregation during solidification of steels. Metallurgical and Materials Transactions A, 2001, 32A: 1755-1767.

[5] Wheeler A A, Boetinger W J, and McFadden G B. Phase field model for isothermal transition in binary alloys. Physical Review $A$, 1992, 45(10): 7424-7439.

[6] Ahmad N A, Wheeler A A, Boettinger W J, et al. Solute trapping and solute drag in a phase field model of rapid solidification. Physical Review E, 1998, 58: 3436-3450.

[7] Loginova I, Amberg G, and Agren J. Phase field simulation of nonisothermal binary alloy solidification. Acta Materialia, 2001, 49: 573-581.

[8] George W L, and Warren J A. A parallel 3D dendritic growth simulator using the phase field method. Journal of Computational Physics, 2002, 177: 264-283.

[9] Toshio Suzuki, Machiko Ode, and Seong Gyoon Kim. Phase-field model of dendritic growth. Journal of Crystal Growth, 2002, 237239: 125-131.

[10] Guo Jingjie, Li Xinzhong, Su Yanqing, et al. Phase-field simulation of structure evolution at high growth velocities during directional solidification of $\mathrm{Ti}_{55} \mathrm{Al}_{45}$ alloy. Intermetallics, 2005, 13: 275-279.

[11] Li Xinzhong, Guo Jingjie, Su Yanqing, et al. Phase-field simulation of dendritic growth for binary alloys with complicated solution models. Transactions of Nonferrous Metals Society of China, 2005, 15(4): 769-776.

[12] Li Xinzhong, Su Yanqing, Guo Jingjie, et al. Phase-field simulation of evolution of interfacial morphology and microstructure for Ti45\%Al alloy. Journal of Central South University, 2006, 37(5): 856-861.

[13] Boettinger W J, Warren J A, Beckermann C, et al. PhaseField Simulation of Solidification. Annual Review of Materials Research, 2002, 32: 163-194. 